\title{
81. DOS NUEVOS HÍBRIDOS DE TEUCRIUM L. (LAMIACEAE)
}

\author{
Pedro SÁNCHEZ GÓMEZ, Antonio F. CARRILLO, Juan F. JIMÉNEZ, \\ Miguel A. CARRIÓN VILCHES, Antonio HERNÁNDEZ y Teresa NAVARRO
}

Two new hybrids of Teucrium L. (Lamiaceae)

Palabras clave. Híbridos, Teucrium, Lamiaceae, Península Ibérica.

Key words. Hybrids, Teucrium, Lamiaceae, Iberian Peninsula.

Teucrium x portusmagnii, P. Sánchez Gómez, Carrillo, A. Hernández \& T. Navarro nothosp. nov.

T. freynii Willk. x T. capitatum subsp. gracillimum (Rouy) Valdés Berm. \& Sánchez Crespo

Holotypus: ESP : Murcia; Cartagena, Atamaria, 30SXG9263, 140m, 16-V-1999, P. Sánchez-Gómez, A. F. Carrillo \& A. Hernández, MGC 48203.

A genitore Teucrium freynii Willk., differt foliis linearis margine revolutis; calycibus $(3,5 \mathrm{~mm})$ tubularibus campanulatis; floribus in racemos dense dispositis in panicula composita; corollis lobis lateralibus posterioribus elongatis cum pilis simplicibus non glanduliferis. A genitore T. capitatum subsp. gracillimum (Rouy) Valdés Berm. \& Sánchez Crespo differt pilis patentibus vel vermiformibus (nec ramosis). (fig. 1)

Este híbrido lleva el nombre de la localidad donde se encuentra, proximidades de Portman (Portus Magnus).

Las hojas son lineares a linear-lanceoladas de márgenes revolutos como en $T$. capitatum subsp. gracillimum y de base ligeramente cuneada como en $T$. freynii; el cáliz es tubularcampanulado, estrecho, ligeramente dorsiventral y con dientes triangular-agudos como en $T$. freynii, pero nunca excede 10 s $3,5 \mathrm{~mm}$ de longitud como en $T$. capitatum subsp. gracillium; la inflorescencia es ramificada y los racimos densos y pequeños como en $T$. capitatum subsp. gracillimum, nunca racimos laxos terminales como en $T$. freynii; la corola presenta los lóbulos latero-posteriores desarrollados y exertos como en $T$. freynii pero de extremos ciliados como en $T$. capitatum subsp. gracillimum y el indumento está constituido de pelos simples no ramificados como en $T$. freynii.

Vive en matorrales sobre litosuelos calcáreos En su comunidad vegetal, son especies acompañantes: Teucrium carthaginenese Lange, Satureja obovata subsp. canescens (Rouy) Rivas Mart., Sideritis pusilla subsp. carthaginensis (Font Quer) Alcaraz et al., Anacamptis pyramidalis (L.) Rich....etc.

Teucrium $x$ guemesii J.F. Jiménez, Carrillo, Carrión Vilches, P. Sánchez-Gómez \& T. Navarro nothosp. nov.

T. carolipaui subsp. fontqueri (Sennen) Rivas Mart. x T. lanigerum Lag.

A genitore Teucrium carolipaui subsp. fontqueri (Sennen) Rivas Mart., differt tota planta puberula; caulibus, foliiis et calycibus pilosis (nec glandulosis); foliis crenatis; DGES.

Trabajo financiado con cargo a los proyectos FLP/9/FS97 de la Fundación Séneca y PB97-1061 de la 

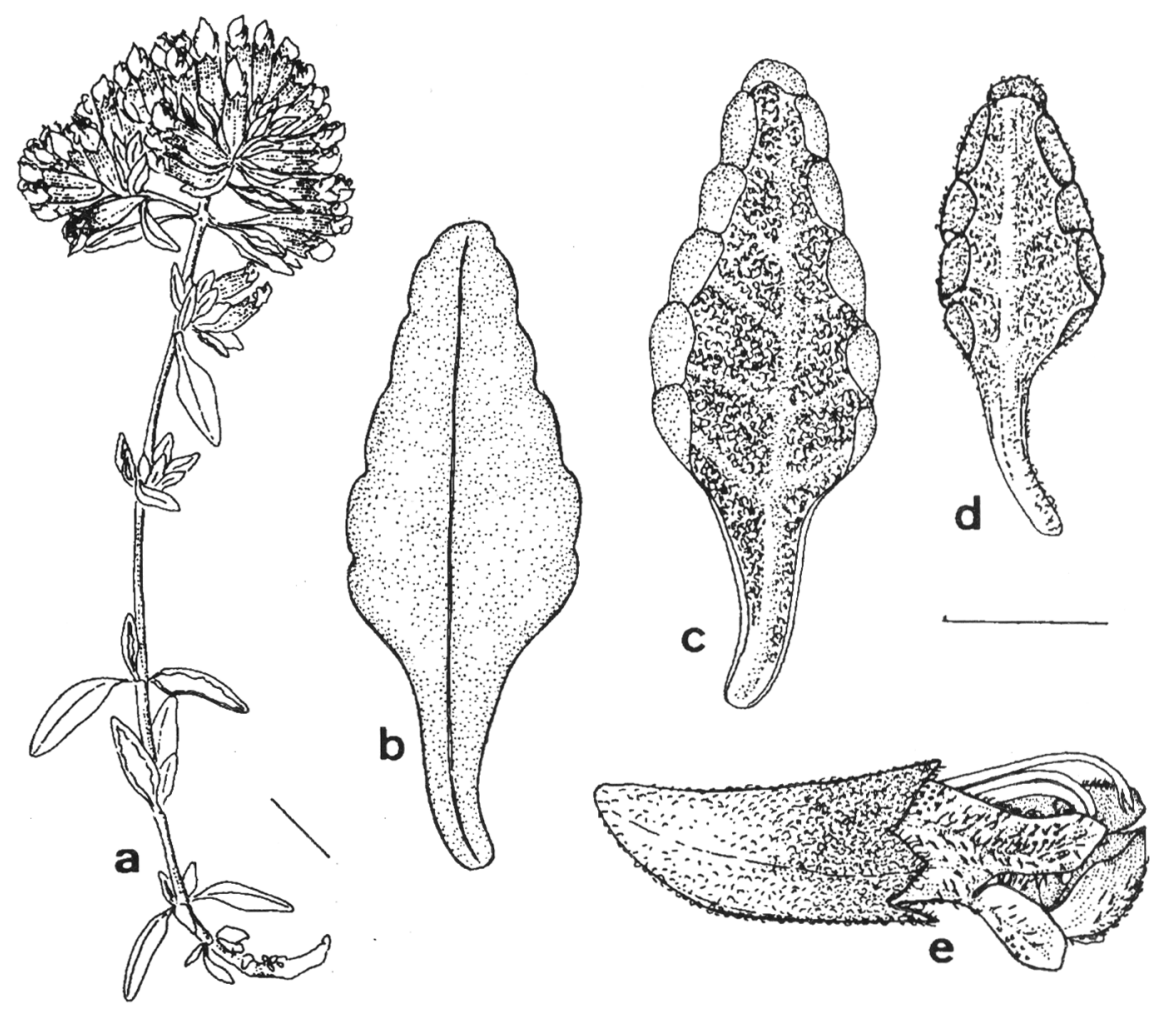

Figura 1. Teucrium x portusmagnii: a. hábito (x 5); b. hoja, cara adaxial; c. hoja, cara abaxial; d. bráctea de la inflorescencia, cara abaxial; e. detalle de la flor (x 2) (escala= $2 \mathrm{~mm}$ ).

floribus in racemos elongatos (nec sphaericos). A genitore Teucrium lanigerum Lag., differt pilis in foliis et calycis sparsis (nec tomentosibus); foliis base linearis (nec subamplexicaulibus); corollis lobis lateralibus posterioribus elongatis, exertis. (Fig. 2).

Holotypus: ESP: Murcia; Aguilas, La Carolina, 30SXG2137, 45m, 4-VI-1999, J.F. Jiménez, A.F. Carrillo \& M.A. Carrión Vilches, MGC 48204.

Este híbrido está dedicado al Dr. Jaime Güemes Heras, botánico levantino.

El tallo, las hojas y cálices son pulverulentos con indumento de pelos simples largos vermiformes más denso en los tallos y en la cara abaxial de las hojas, nunca villoso ni densotomentoso como en $T$. lanigerum. Las hojas son linear-lanceoladas como en $T$. carolipaui subsp. fontqueri, pero ligeramente crenadas a partir de la mitad superior como en $T$. lanigerum, pero nunca subamplexicaules ni erecto-ascendentes. Cáliz tubular, estrecho de dientes triangulares agudos largos como T. carolipaui subsp. fontqueri, pero pulverulento de pelos simples largos vermiformes con ausencia de pelos glandulares séxiles. Inflorescencia en racimos largos espiciformes como $T$. lanigerum, nunca esferico-subcónicos como en $T$. carolipaui subsp. fontqueri. Corola de lóbulos laterales y latero-posteriores desarrollados 

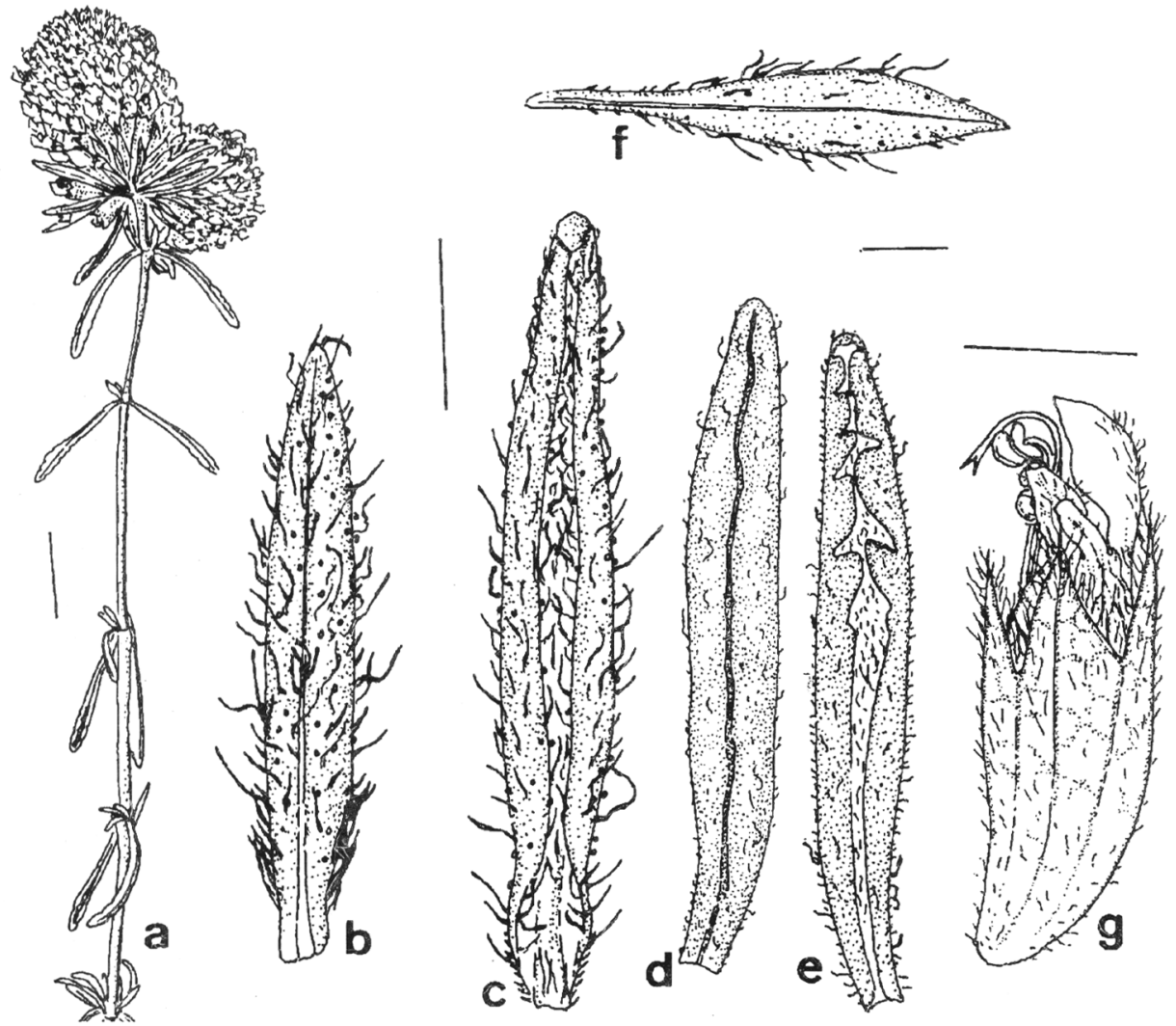

Figura 2. Teucrium x guemesii: a. hábito (escala $=8 \mathrm{~mm}$ ); b. hoja, cara adaxial; c. hoja, cara abaxial (x 10, escala $=2 \mathrm{~mm}$ ); d. bráctea de la inflorescencia, cara adaxial; e. bráctea de la inflorescencia, cara abaxial; f. bracteola, cara adaxial $(x 5$, escala $=2 \mathrm{~mm})$; g. detalle de la flor $($ escala $=2 \mathrm{~mm})$.

como T. carolipaui subsp. fontqueri.

Vive entre los padres en matorrales de caméfitos sobre suelos pedregosos y margosos Son especies acompañantes de su comunidad: Teucrium murcicum Sennen, Santolina viscosa Lag., Fumana ericoides (Cav.) Gand., Sideritis ibanyezii $\mathrm{Pau}$, Launaea arborescens (Batt.) Murb., Asteriscus maritimus (L.) Less., Lygeum spartium L...etc.
Aceptado para su publicación en Octubre de 1999

Dirección de los autores. P. SÁNCHEZ GÓMEZ, A.F. CARRILLO, J. F. JIMÉNEZ, M. A. CARRIÓN VILCHES y A. HERNÁNDEZ: Dpto. Biología Vegetal (Botánica). Fac. de Biología. Campus del Espinardo. 30100, Murcia.e-mail: psgomez@fcu.um.es; T. NAVARRO: Dpto. Biología Vegetal. Fac. de Ciencias. Universidad de Málaga. Campus de Teatinos. 29071, Málaga. E-mail: tnavarro@uma.es. 